\title{
66. Über eine neue Methode zur Registrierung des Aktionsstromes des Herzens.
}

\author{
Das fortlaufende Vektordiagramm mit der Zeitmarkierung.)
}

Von Seizo Katsunuma und Hideo Toyoshima.

I. Medizinische'Klinik der Kaiserl. Universität, Nagoya.

(Comm. by K. MiurA, M.I.A., June 12, 1943.)

Aúf dem Wiesbadener Kongress 1936 .hat Schellong über eine Methode berichtet, mit der man die Potentialdifferenzen zweiter Ableitungen des menschlichen Elektrokardiogramms (I. und III. Ableitung) durch direktes Registrieren mit der Braunschen Röhre $\mathrm{zu}$ einer Figur zusammensetzen kann, die der vektoriellen Summe der in diesen beiden Ableitungen vorhandenen Potentialdifferenzen entspricht. Er bezeichnet diese Figur als Vektordiagramm.

Um die räumlichen Verhältnisse der Aktionsströme des Herzens darzustellen, benutzte er die stereoskopische Betrachtung des aus zwei verschiedenen Ebenen abgeleiteten Vektordiagramms, das aus einer frontalen Ebenen auf die rechte Seite und das aus einer schrägeren Ebene auf die linke Seite legend.

Das auf diese Weise gewonnene Vektordiagramm besteht aus drei verschiedenen Schleifen und zwar der P-, QRS- und T-Schleife, und es stellt die räumliche Vorstellung dar. Nach der Form, den raumlichen Verhältnissen und der Umlaufsrichtung dieser Schleifen kann man die funktionellen oder organischen Veränderungen des Herzens beurteilen. Wir haben uns auch seit drei Jahren mit der Schellongschen Methode beschäftigt und erkannten, wie aus unseren früheren Berichten (siehe Mitt. Med. Ges. Nagoya, Bd. 57, 1941) hervorgeht, ihre grosse Bedeutung. Da es aber schwer ist, das Vektordiagramm aufeinanderfolgend synchron mit den Herzschlägen zu photographieren, hatten wir grosse Schwierigkeit bei der fortlaufenden Betrachtung der Extrasystole oder anderer Arhythmien.

Aber wir konnten diese Schwierigkeit leicht beseitigen, ohne die räumlichen Verhältnisse zu stören, indem wir den Film vermittels eines von uns hergestellten Apparates mit konstanter Geschwindigkeit langsam von oben nach unten laufen liessen. Die nach dieser Methode registrierte Kurve besteht aus drei Spiralen, der P-, QRS- und T-Spirale und alle verlaufen um die Nulllinie spiral im Sinne des Uhrzeigers oder umgekehrt. Die Spirale entspricht jeder für sich der P., QRSund T-Zacke im Elektrokardiogramme oder der P-, QRS- und T-Schleife im Vektordiagramme. Auf dieser Kurve kann man sowohl die rhythmischen wie auch die raumlichen und zeitlichen Verhälnisse des Aktionsstromes des Hërzens beobachten. Schwierig war beim Vektordiagramme auch die Differenzierung der P- und T-Schleife, wegen ihres Zusammenfallens mit dem Nullpunkt. Nach unserer Methode aber lasst sich die P- und T-Schliefe leicht differenzieren, ohne die raumliche Vorstellung 
zu verlieren und man kann auch leicht mit der bisherigen Methode vergleichen, wenn man unseren Apparat anwendet. Wir möchten das Vektordiagramm nach unserer Methode vorläufig als „Vektorelektrokardiogramm " bezeichnen.

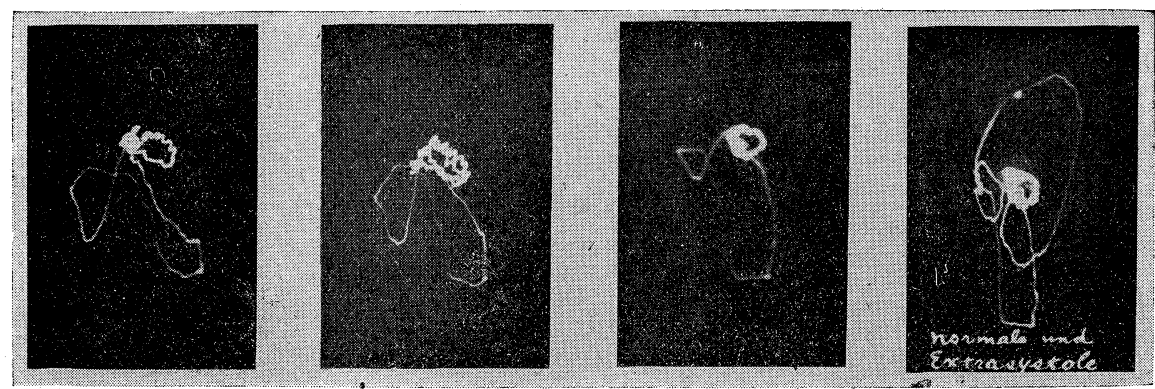

Abb. 1.

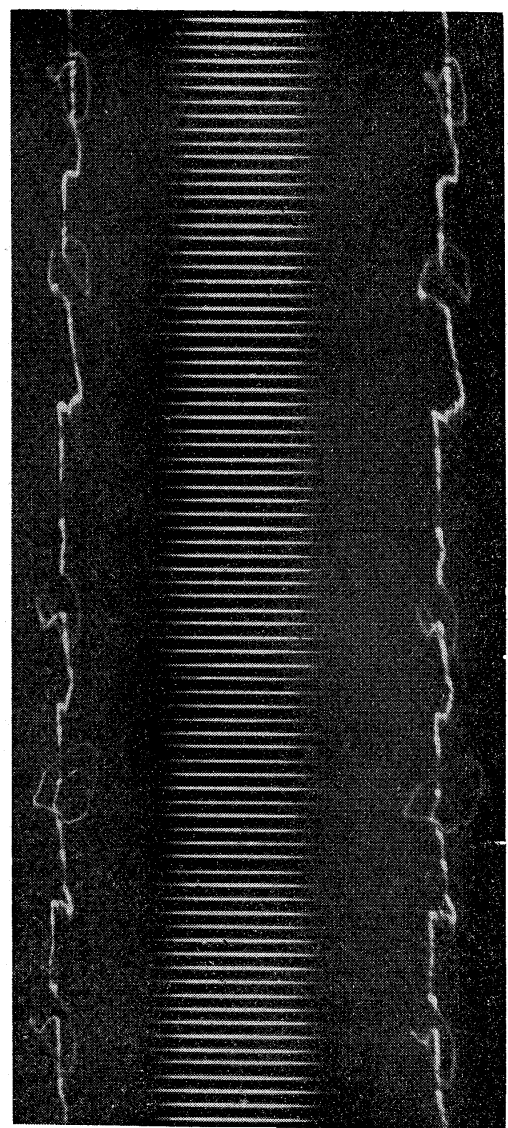

Abb. 2.

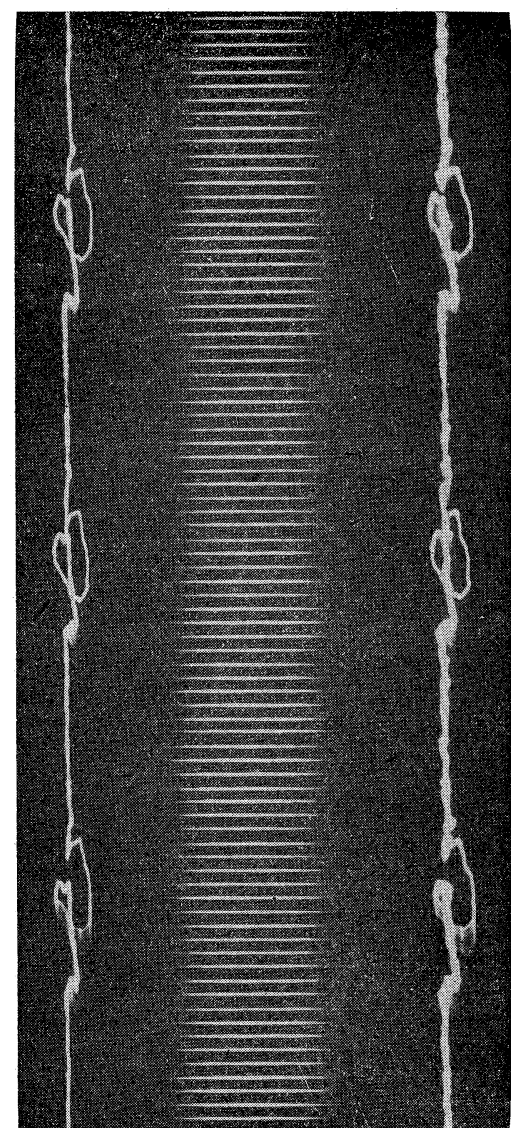

Abb. 3.

Abb. 1 zeigt das Vektordiagramm eines Kranken mit Arhythmia Perpetua aus frontaler Ebene. Aus dieser Figur ist zu ersehen, dass bei der Arhythmie der Kammerkomplex in verschiedener Form und Umlaufsrichtung auftritt. Aber diese Figur ermöglicht es uns nicht, 
No. 6.] Über eine neue Methode zur Registrierung des Aktionsstromes.

etwas überr die rhythmischen und zeitlichen Verhältnisse des Herzens $\mathrm{zu}$ sagen.

Abb. 2 zeigt das Vektorelektrokardiogramm desselben Kranken wie Abb. 1 nach unserer Methode. Nàch dieser Kurve kann man die rhythmischen, räumlichen und auch zeitlichen Verhältnisse erörtern.

Abb. 3 ist das Vektorelektrokardiogramm eines Gesunden. 